\title{
Insufficient evidence for effectiveness of any treatment for oral lichen planus
}

\author{
Abstracted from \\ Thongprasom K, Carrozzo M, Furness S, Lodi G. \\ Interventions for treating oral lichen planus. Cochrane Database Syst Rev 2011; Issue 7 \\ Address for correspondence: Luisa Fernandez Mauleffinch, \\ Review Group Co-ordinator, Cochrane Oral Health Group, MANDEC, School of Dentistry, \\ University of Manchester, Higher Cambridge Street, Manchester, M15 6FH, UK. \\ E-mail: luisa.fernandez@manchester.ac.uk
}

\section{Question: What is the safest and most effective treatment for symptomatic oral lichen planus (OLP)?}

Data sources The Cochrane Oral Health Group Trials Register, Cochrane Central Register of Controlled Trials (CENTRAL), Medline and Embase databases were searched with no restrictions regarding language or date of publication. Bibliographies of identified publications were also checked for relevant studies, and authors were contacted to identify missing and unreported trials. The WHO International Trials Registry Platform was searched for ongoing studies, using the term 'oral lichen planus'.

Study selection Randomised controlled clinical trials (RCTs) of therapy for symptomatic OLP which compared treatment with a placebo or between treatments or no intervention were considered for this review.

Data extraction and synthesis The titles and abstracts of all reports identified were scanned independently by two review authors. All studies meeting the inclusion criteria were assessed for risk of bias and data were extracted. For dichotomous outcomes, the estimates of effects for the intervention were expressed as risk ratios (RR) together with $95 \%$ confidence intervals. For continuous outcomes, mean differences (MD) and 95\% confidence intervals were used to summarise the data. Meta-analyses were conducted for studies with similar comparisons reporting the same outcome measures.

Results Twenty-eight trials were included. Although topical steroids are considered first line treatment for symptomatic OLP, we identified no RCTs that compared steroids with placebo. There is no evidence from the three trials of pimecrolimus that this treatment is better than placebo in reducing pain from OLP. There is weak evidence from two trials, at unclear and high risk of bias respectively, that aloe vera may be associated with a reduction in pain compared to placebo, but it was not possible to pool the pain data from these trials. There is weak and unreliable evidence from two small trials, at high risk of bias, that cyclosporin may reduce pain and clinical signs of OLP, but metaanalysis of these trials was not possible.

This paper is based on a Cochrane Review published in the Cochrane Library 2011, issue 7 (see www.thecochranelibrary.com for information). Cochrane Reviews are regularly updated as new evidence emerges and in response to feedback, and the Cochrane Library should be consulted for the most recent version of the review.
There were five trials that compared steroids with calcineurin inhibitors, each evaluating a different pair of interventions. There is no evidence from these trials that there is a difference between treatment with steroids compared to calcineurin inhibitors with regard to reducing pain associated with OLP. From six trials there is no evidence that any specific steroid therapy is more or less effective at reducing pain compared to another type or dose of steroid.

Conclusions From the 28 trials included in this systematic review, the wide range of interventions compared means there is insufficient evidence to support the effectiveness of any specific treatment as being superior.

\section{Commentary}

Effective palliative treatment of oral lichen planus is very important for patients and clinicians. Oral lichen planus (OLP) is a chronic autoimmune disease. There are a number of types, but the atrophic and erosive type can cause symptoms of burning to severe pain and remission is rare. Quality of life is severely affected. ${ }^{1}$ Steroids in different varieties, formulations and concentrations were for years the gold standard for treatment. Now there are many products on the market that claim they can be as useful as corticosteroids and perform the treatment without the side effects of the steroids. This systematic review considered studies evaluating the use of any OLP treatment comparing an active treatment with placebo or with another active treatment. Pain reduction was the main outcome assessed by the patient.

An appropriate number of databases and publications in English language only were searched and reported by the authors. Although it was stated that non-English language review was performed there was no documentation of those databases being searched.

This very extensive review undoubtedly analysed the data efficiently to provide answers to the question, appropriately selected the right studies (randomised clinical trials for therapy questions) and assessed the quality of the individual studies to minimise the risk of bias. Meta-analysis was not possible for all the combined 28 studies due to high heterogeneity. Meta-analysis was done for some of the similar studies evaluating the same drug for treatments. An important feature of the review was the calculation of the drop-out rate and the intention to treat analysis. There was no more than a $20 \%$ drop-out rate in all the 28 studies. The patients in 11 trials completed the study, seven trials had a 10\% drop out rate and nine had between 10 and 20\%. Due to small sample size in each study there is a lack of power to detect a significant difference. 
From the evidence presented by the review and the analysis of two trials at high risk of bias it seems that aloe vera may be effective in the reduction of pain. At the same time no evidence was established for the effectiveness of pimecrolimus and very weak evidence shows that cyclosporine may be effective in reducing pain. From the trials comparing different steroids treatments there was no evidence that any steroid is more effective than another or any significant differences in trials that compared steroids with calcineurim inhibitors.

Also, a RCT published but not yet included since it is still in review shows improvement with the use of lasers without the side effects that come with steroid use. ${ }^{2}$

Another study published later comparing Triamcinolone versus aloe vera showed that aloe vera reduced the symptoms of pain and burning sensation equal to the triamcinolone without side effects. ${ }^{3}$

At the present time there is no cure for lichen planus, but emerging therapies are being evaluated with new lines of medications and laser treatments. We, as practitioners, need to keep a watchful eye on future research - palliative as well as curative.

\section{Practice points}

- Practitioners need to be aware that there appear to be new treatments on the horizon which may impact the palliative treatment of lichen planus as well as steroids do but without the side effects.

\section{Analia Veitz Keenan and Debra Ferraiolo}

NYU College of Dentistry, New York, USA

1. Lopez-Jornet P, Camacho-Alonso F. Quality of life in patients with oral lichen planus. Eval Clin Pract 2010; 16: 111-113.

2. Jajarm HH, Falaki F, Mahdavi O. A comparative pilot study of low intensity laser versus topical corticosteroids in the treatment of erosive-atrophic oral lichen planus. Photomed Laser Surg 2011; 29: 421-425.

3. Mansourian A, Momen-Heravi F, Saheb-Jamee M, Esfehani M, Khalilzadeh $\mathrm{O}$, Momen-Beitollahi J. Comparison of treatment efficacy of daily use of aloe vera mouthwash with triamcinolone acetonide $0.1 \%$ on oral lichen planus: A randomized double-blinded clinical trial. Am J Med Sci 2011; Jun 15 (Epub ahead of print)

Evidence-Based Dentistry (2011) 12, 85-86. doi:10. 1038/sj.ebd.6400814 